Communications in Physics, Vol.31, No. 1 (2021), pp. 67-76

DOI:10.15625/0868-3166/15377

\title{
COMPRESSION EFFECTS ON STRUCTURAL RELAXATION PROCESS OF AMORPHOUS INDOMETHACIN
}

\author{
TRAN DINH CUONG ${ }^{1, \dagger}$ AND ANH D. PHAN ${ }^{1,2, \ddagger}$ \\ ${ }^{1}$ Phenikaa Institute for Advanced Study (PIAS), Phenikaa University, Hanoi 12116, Vietnam \\ ${ }^{2}$ Faculty of Computer Science, Materials Science and Engineering, Artificial Intelligence \\ Laboratory, Phenikaa University, Hanoi 12116, Viet Nam \\ E-mail: †cuong.trandinh@phenikaa-uni.edu.vn; `anh.phanduc@phenikaa-uni.edu.vn
}

Received August 15, 2020

Accepted for publication 16 October 2020

Published 8 January 2021

\begin{abstract}
Indomethacin is a common nonsteroidal anti-inflammatory drug, but its glass transition behaviors remain ambiguous. Here we present a simple theoretical approach to investigate the molecular mobility of amorphous indomethacin under compression. In our model, the relaxation of a particle is governed by its nearest-neighbor interactions and long-range cooperative effects of fluid surroundings. On that basis, the temperature and pressure dependence of the structural relaxation time is deduced from the thermal expansion process. Additionally, we also consider correlations between the activated dynamics and the shear response in the deeply supercooled state. Our numerical calculations agree quantitatively well with previous experimental works.
\end{abstract}

Keywords: compression effects, structural relaxation, amorphous drug, indomethacin.

Classification numbers: 65.60.+a.

\section{INTRODUCTION}

Indomethacin (IMC) has been intensively studied for decades because of its peculiar properties [1-3]. This nonselective cyclooxygenase inhibitor is useful for treating joint diseases [4], headaches [5], and patent ductus arteriosus [6]. In addition, one can apply IMC to design smart probes for identifying cancer cells [7-9]. However, the commercial form of IMC exhibits poor aqueous solubility and low bioavailability [10]. Hence, a large dosage of IMC must be delivered to patients to achieve the therapeutic effect. The event may increase the risk of gastrointestinal toxicity [11]. One of the promising strategies to overcome these drawbacks is to prepare IMC in the amorphous form [12-14]. Unfortunately, the disordered atomic structure is thermodynamically 
unstable. The crystallization readily occurs during manufacturing, storage, or dissolution [12-14]. Consequently, precise knowledge of relaxation processes in IMC is essential for reinforcing its physical stability.

In experiments, the structural relaxation time, $\tau_{\alpha}$, is typically measured by the broadband dielectric spectroscopy (BDS) technique [14-16]. The global atomic rearrangement causes a prominent, broad, and asymmetry $\alpha$-peak on the dielectric loss spectra. From these, one can capture a dramatic growth of $\tau_{\alpha}$ upon isobaric cooling or isothermal squeezing [14-16]. When $\tau_{\alpha}$ reaches $10^{2} \mathrm{~s}$, amorphous pharmaceutical systems undergo the glass transition and fall out of equilibrium. In the glassy state, since cooperative motions become frozen, $\tau_{\alpha}$ is often indirectly estimated by a master plot construction [14]. Notably, recent BDS studies [17] have revealed that compression effects can suppress the strong crystallization tendency of IMC. Nevertheless, the underlying mechanism of these phenomena has remained ambiguous.

Apart from BDS measurements, one can use the Elastically Collective Nonlinear Langevin Equation (ECNLE) theory to gain insights into the molecular mobility of amorphous drugs [1820]. The ECNLE theory considers the $\alpha$-relaxation as a dynamic coupling between local and collective activated events [18-20]. On that basis, the thermal response of $\tau_{\alpha}$ is determined by analyzing the bulk expansion process [18-20]. At ambient pressure, ECNLE calculations can provide $\tau_{\alpha}$ from $10^{-12}$ to $10^{2} \mathrm{~s}$, which is far beyond the simulation timescale [18-20]. Recently, Phan et al. [21,22] have modified the free energy profile to access the high-pressure regime. The obtained results for $\tau_{\alpha}$ of Phan et al. [21,22] are in good agreement with BDS data. However, the modified ECNLE model [21,22] may lead to incorrect predictions of the dynamic fragility.

In this paper, we extend the ECNLE analysis to solve the mentioned limitations. Numerical calculations are carried out for IMC up to $226 \mathrm{MPa}$. Besides, correlations between the structural relaxation time, the fragility index, and the instantaneous shear modulus are comprehensively discussed. Our theoretical results are quantitatively compared with prior experiments.

\section{THEORETICAL BACKGROUND}

In the ECNLE theory [18-22], an amorphous drug is described by an effective hard-sphere fluid having the particle diameter, $\sigma$, the particle number density, $\rho$, and the packing fraction, $\Phi=\pi \rho \sigma^{3} / 6$. Structural properties of the reference system are inferred from the Percus-Yevick approximation [23]. Specifically, the direct correlation function, $C(r)$, is written in the real-space by

$$
\begin{aligned}
C(r) & =-\frac{(1+2 \Phi)^{2}}{(1-\Phi)^{4}}+\frac{3 \Phi(2+\Phi)^{2}}{2(1-\Phi)^{4}} \frac{r}{\sigma} \\
& -\frac{\Phi(1+2 \Phi)^{2}}{2(1-\Phi)^{4}}\left(\frac{r}{\sigma}\right)^{3} \text { for } r \leq \sigma, \\
C(r) & =0 \text { for } r>\sigma .
\end{aligned}
$$

Employing the Fourier transform yields

$$
C(k)=\frac{4 \pi}{k} \int_{0}^{\sigma} C(r) \sin (k r) r d r,
$$




$$
\begin{gathered}
S(k)=\frac{1}{1-\rho C(k)}, \\
g(r)=1+\frac{1}{2 \pi^{2} \rho r} \int_{0}^{\infty}[S(k)-1] k \sin (k r) d k,
\end{gathered}
$$

where $k$ is the wavevector, $S(k)$ is the static structure factor, and $g(r)$ is the radial distribution function.

Molecular mobility is investigated in the framework of slow dynamics. In the overdamped limit, stochastic trajectories of the tagged particle are governed by the nonlinear Langevin equation as $[24,25]$

$$
-\zeta_{s} \frac{\partial r}{\partial t}-\frac{\partial F_{d y n}}{\partial r}+\delta f=0
$$

where $r$ is the scalar displacement, $\zeta_{s}$ is the short-time friction constant, $\delta f$ is the thermal white noise, and $F_{d y n}$ is the dynamic free energy derived from nearest-neighbor interactions. The explicit form of $F_{d y n}$ is $[24,25]$

$$
\frac{F_{d y n}}{k_{B} T}=-3 \ln \frac{r}{\sigma}-\int \frac{d \mathbf{k}}{(2 \pi)^{3}} \rho C^{2}(k) S(k)\left[1+S^{-1}(k)\right]^{-1} \exp \left\{-\frac{k^{2} r^{2}}{6}\left[1+S^{-1}(k)\right]\right\},
$$

where $k_{B}$ is Boltzmann's constant and $T$ is temperature. The first term in Eq. (7) describes the ideal fluid state, while the second term represents an entropic trapping potential $[24,25]$.

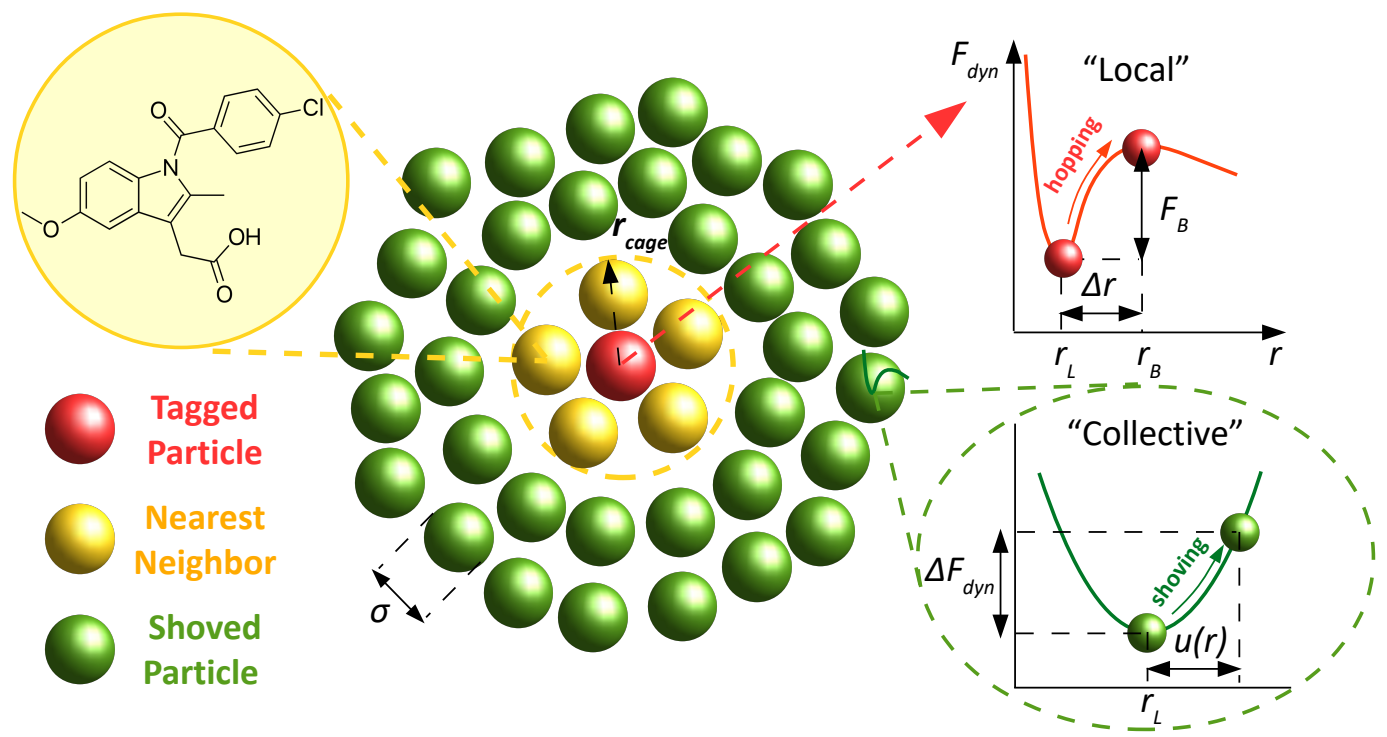

Fig. 1. (Color online) Illustrations of the ECNLE theory for the $\alpha$-relaxation process. Each IMC molecule is modeled by an effective hard-sphere.

As shown in Figure 1, $F_{d y n}$ gives us basic information about local dynamics. In dilute solutions $(\Phi<0.43), F_{d y n}$ reduces monotonically with increasing $r[24,25]$. This tendency reveals 
that fluid particles can diffuse without kinetic constraints. For $\Phi>0.43$, a finite potential well emerges from $F_{d y n}[24,25]$. Hence, the tagged particle is dynamically arrested within an intermolecular cage. The cage radius, $r_{\text {cage }}$, is determined by the first minimum of $g(r)$ [18-22]. Based on the free energy profile, one obtains three important length scales, including the localization length, $r_{L}$, the barrier position, $r_{B}$, and the jump distance, $\Delta r$. The local barrier is defined by $F_{B}=F_{d y n}\left(r_{B}\right)-F_{d y n}\left(r_{L}\right)$.

In the localized state, the instantaneous shear modulus, $G_{\infty}$, can be deduced from the GreenKubo formula, which is [26,27]

$$
G_{\infty}=\frac{k_{B} T}{60 \pi^{2}} \int_{0}^{\infty} d k\left[\frac{k^{2}}{S(k)} \frac{d S(k)}{d k}\right]^{2} \exp \left[-\frac{k^{2} r_{L}^{2}}{3 S(k)}\right] .
$$

At high densities, since $r_{L} \ll \sigma$, only large wavevectors contribute to $G_{\infty}$. This ultra-local analysis [28] allows us to compact Eq. (8) by

$$
G_{\infty}=\frac{9}{5 \pi} \frac{k_{B} T \Phi}{\sigma r_{L}^{2}} .
$$

The activated hopping event of the tagged particle requires the rearrangement of its nearest neighbors [29-31]. Therefore, one can observe a small increase in the cage's volume. This local dilation excites a harmonic displacement field, $u(r)$, in the surrounding fluid. Utilizing Lifshitz's continuity equation [32] provides

$$
u(r)=\Delta r_{\text {eff }}\left(\frac{r_{\text {cage }}}{r}\right)^{2}, \quad r \geq r_{\text {cage }},
$$

where $\Delta r_{\text {eff }} \approx 3 \Delta r^{2} / 32 r_{\text {cage }}$ is the cage expansion amplitude [29-31].

According to Einstein's glass picture, fluid particles outside the cage vibrate with the same harmonic force constant, $K_{L}=\left(\partial^{2} F_{d y n} / \partial r^{2}\right)_{r=r_{L}}$. Consequently, the strain energy, known as the collective elastic barrier, is computed by [29-31]

$$
F_{E}=\int_{r_{\text {cage }}}^{\infty} \frac{1}{2} K_{L} u^{2}(r) \rho g(r) 4 \pi r^{2} d r \approx 12 \Phi \Delta r_{e f f}^{2}\left(\frac{r_{\text {cage }}}{\sigma}\right)^{3} K_{L},
$$

where $g(r) \approx 1$ for $r \geq r_{\text {cage }}$. From Eqs. (9) and (11), one can associate $F_{E}$ with $G_{\infty}$ at the deeplysupercooled regime by

$$
F_{E}=20 \pi \frac{\Delta r_{e f f}^{2} r_{\text {cage }}^{3}}{\sigma^{2}} G_{\infty}
$$

Figure 2 shows how $F_{B}$ and $F_{E}$ depend on the packing fraction. Essentially, one can neglect $F_{E}$ in a range of $0.43<\Phi<0.55$. Nevertheless, in dense systems $(\Phi>0.57), F_{E}$ grows much faster than its local analog. Entropic barrier profiles cross each other at $\Phi \approx 0.6$. This result affirms the dominance of collective dynamics in the $\alpha$-relaxation near kinetic vitrification [29-31].

Recall that $\tau_{\alpha}$ is defined as the average time for the tagged particle to escape from its cage. Adopting the modified Kramers' theory yields [29-31]

$$
\tau_{\alpha}=\tau_{s}\left[1+\frac{2 \pi}{\sqrt{K_{L} K_{B}}} \frac{k_{B} T}{\sigma^{2}} \exp \left(\frac{F_{B}+F_{E}}{k_{B} T}\right)\right],
$$


where $K_{B}=-\left(\partial^{2} F_{d y n} / \partial r^{2}\right)_{r=r_{B}}$ is the absolute curvature at the barrier position, and $\tau_{s}$ is the short relaxation timescale. The analytical expression of $\tau_{s}$ is [29-31]

$$
\begin{gathered}
\tau_{s}=\tau_{E} g^{2}(\sigma)\left\{1+\frac{\sigma^{3}}{36 \pi \Phi} \int_{0}^{\infty} d k \frac{k^{2}[S(k)-1]^{2}}{S(k)+b(k)}\right\}, \\
b^{-1}(k)=1-j_{0}(k \sigma)+2 j_{2}(k \sigma),
\end{gathered}
$$

where $\tau_{E} \approx 10^{-13} \mathrm{~s}$ is the Enskog timescale, $g(\sigma)$ is the contact value of the radial distribution function, and $j_{n}(x)$ is the spherical Bessel function of order $n$.

Non-Arrhenius behaviors of $\tau_{\alpha}$ are reflected in the dynamic fragility, which is [14]

$$
m=\left[\frac{\partial \log _{10} \tau_{\alpha}}{\partial\left(T_{g} / T\right)}\right]_{T=T_{g}},
$$

where $T_{g}$ is the glass transition temperature. By using the fragility index, one can classify amorphous drugs into three main categories: "strong" $(m \leq 30)$, "intermediate" $(0<m<30)$, and "fragile" $(m \geq 100)$ [14].

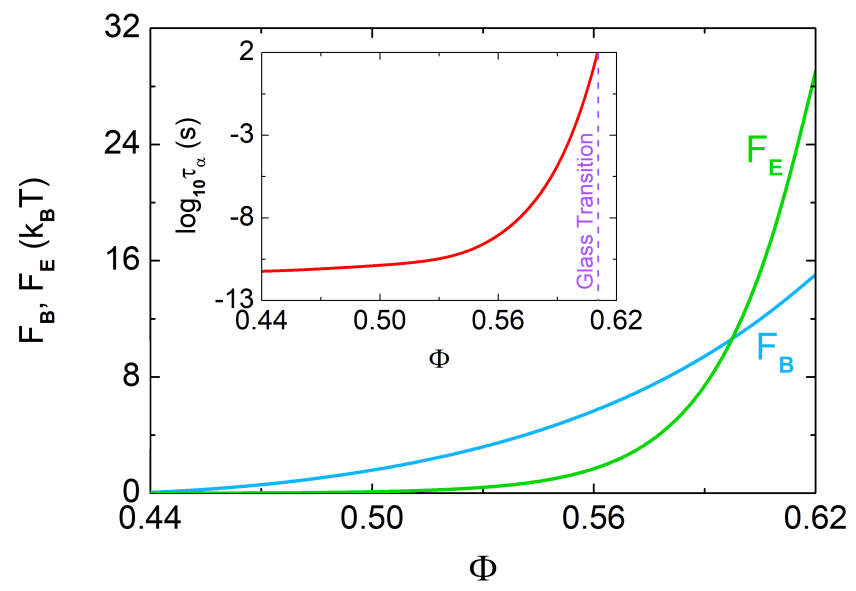

Fig. 2. (Color online) Effects of the packing fraction on entropic barriers (mainframe) and the structural relaxation time (inset) inferred from the ECNLE theory.

As depicted in the inset of Figure 2, the ECNLE theory gives us a universal correlation between the $\alpha$-relaxation and the packing fraction over 14 decades [29]. Thus, a chemical mapping, $\Phi=f(P, T)$, is required to compare our coarse-grained calculations with experiments [30,31]. Schweizer et al. $[30,31]$ have constructed $f(0, T)$ by equating the Percus-Yevick compressibility $(S(k=0))$ to its experimental counterpart. The strategy has been successfully applied to thermal liquids [30] and polymer melts [31]. Unfortunately, numerous amorphous drugs have no equation-of-state data for Schweizer's mapping [30,31]. To handle this issue, Phan et al. [18-22] have proposed an alternative form of $f(0, T)$ via the thermal expansion process. In the present 
work, we extend Phan's mapping [18-22] to appropriately take into account compression effects. The $f(P, T)$ function is written by

$$
\Phi=f(P, T)=\Phi_{0}\left\{1-\beta(P)\left[T-T_{0}(P)\right]\right\},
$$

where $P$ is hydrostatic pressure, $\Phi_{0}$ is the initial packing fraction, $\beta$ is the bulk thermal expansivity, and $T_{0}$ is the characteristic temperature. Remarkably, in most cases, the original Schweizer's mapping [30,31] can be well fitted by Eq. (17) with $\Phi_{0}=0.5$ [27]. We keep utilizing this common value to consider the glassy dynamics of IMC.

Material-specific details (e.g., molar mass and particle size) are encoded in $T_{0}$ [18-22]. Since Eq. (13) indicates $\tau_{\alpha}=10^{2} \mathrm{~s}$ at $\Phi=\Phi_{g}=0.611$, we can directly link $T_{0}$ to $T_{g}$ by

$$
T_{0}=T_{g}+\frac{\Phi_{g}-\Phi_{0}}{\beta \Phi_{0}}
$$

For simplicity, $T_{g}$ is taken from experiments. The obtained results are often described by

$$
T_{g}=k_{1}\left(1+\frac{k_{2}}{k_{3}} P\right)^{1 / k_{2}},
$$

where $k_{1}, k_{2}$, and $k_{3}$ are Andersson's parameters [33]. On that basis, the pressure dependence of $\beta$ is supposed to be [34-36]

$$
\beta=\beta_{0}\left(1+\frac{k_{2}}{k_{3}} P\right)^{-1},
$$

where $\beta_{0}=12 \times 10^{-4} \mathrm{~K}^{-1}$ is employed to all organic materials [18-22].

\section{RESULTS AND DISCUSSION}

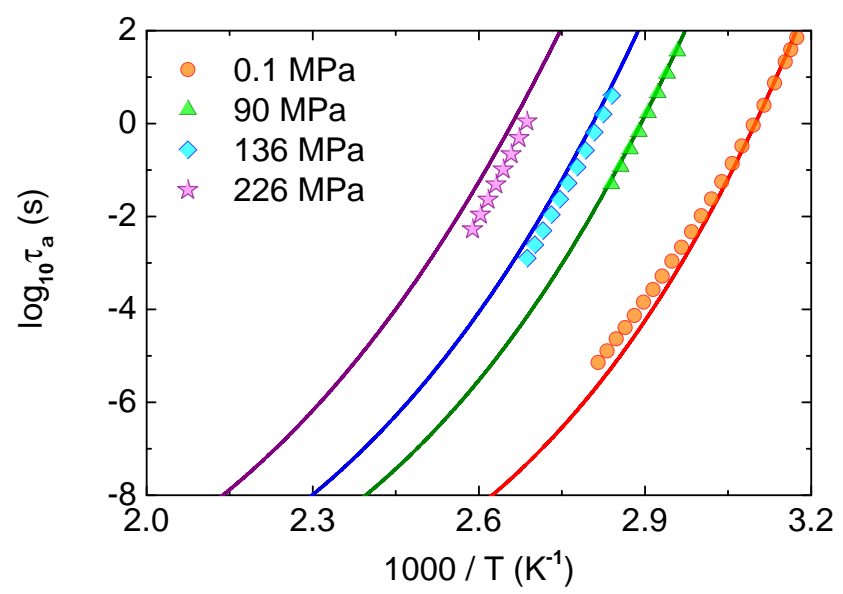

Fig. 3. (Color online) Effects of temperature on the structural relaxation time of IMC at various pressures. Filled points are BDS data in Ref. [17], and solid lines correspond to our ECNLE calculations. 
Figure 3 shows how $\log _{10} \tau_{\alpha}$ of IMC depends on $1000 / T$ at different pressures. Andersson's parameters used for theoretical calculations are $k_{1}=315 \mathrm{~K}, k_{2}=3.14$, and $k_{3}=1238$ MPa [17]. Notably, a large value of $k_{1} k_{3}^{-1}=0.254 \mathrm{~K} \cdot \mathrm{MPa}^{-1}$ suggests that IMC should be viewed as a typical van der Waals liquid [37-43]. From these, we can capture a significant slowing down of molecular dynamics upon isobaric cooling. The ECNLE analysis is in excellent agreement with recent BDS measurements [17]. Consequently, our findings would be useful for the tableting process of amorphous drugs.

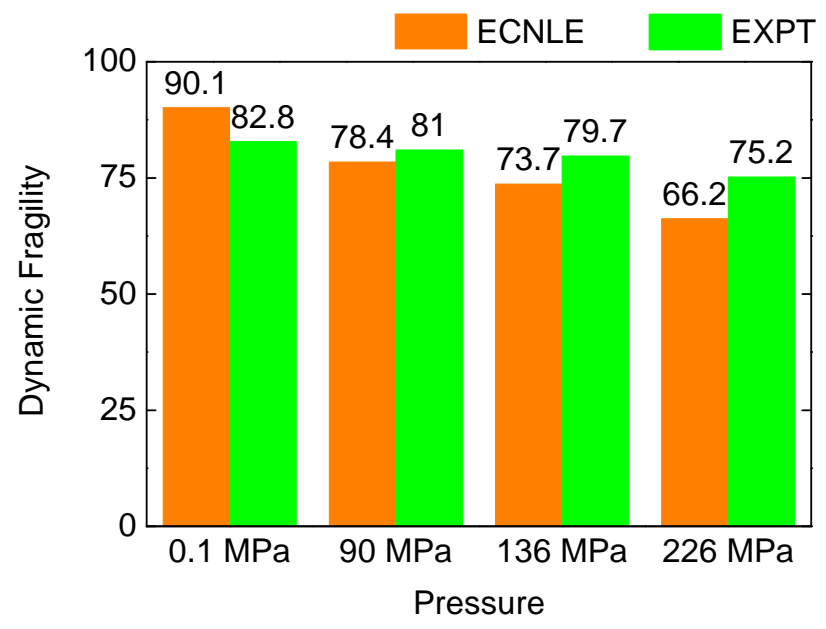

Fig. 4. (Color online) The dynamic fragility of IMC versus pressure given by the ECNLE theory and BDS experiments [17].

Figure 4 shows compression effects on the dynamic fragility of IMC. When $P=0.1 \mathrm{MPa}$, the ECNLE theory predicts $m=90.1$. Thus, IMC is regarded as an "intermediate" glass-forming liquid [14]. Similar to other polymers and van der Waals systems [41-44], IMC becomes "stronger" at elevated pressure. By combining Eqs. (16) and (17), we obtain

$$
m=\beta T_{g} \Phi_{0}\left(\frac{\partial \log _{10} \tau_{\alpha}}{\partial \Phi}\right)_{\Phi=\Phi_{g}} \propto \frac{1}{\left(1+\frac{k_{2}}{k_{3}} P\right)^{\frac{k_{2}-1}{k_{2}}}} .
$$

Since $k_{2}>1$, Eq. (21) clearly explains why $m$ reduces continuously with compression. The linear relation $m \propto \beta T_{g}$ has been confirmed by many literatures [41-44]. Overall, ECNLE results are slightly smaller than BDS data [17]. This discrepancy may be due to the ignorance of hydrogen bonds [14]. However, the maximum error for $m$ is only $12 \%$ in a range of 0 to $226 \mathrm{MPa}$. The value validates our theoretical approach and the chosen chemical mapping.

In the deeply supercooled state, the $\alpha$-relaxation depends crucially on mechanical properties [45-47]. Conventionally, one can use Dyre's shoving parameter, $X$, to clarify correlations between the activated dynamics and the shear response [45-47]. The definition of $X$ is [45-47]

$$
X=\frac{G_{\infty}(P, T) T_{g}}{G_{\infty}\left(P, T_{g}\right) T} .
$$


Applying the ultra-local analysis (Eq. (9)) [28] and an exponential law $r_{L}=30 \sigma \exp (-12.2 \Phi)$ [24] provides

$$
X=\frac{\Phi \exp (24.4 \Phi)}{\Phi_{g} \exp \left(24.4 \Phi_{g}\right)} .
$$

Numerical calculations based on Eqs. (17) and (23) are presented in Figure 5. For IMC, one can realize a dramatic growth of Dyre's shoving parameter with decreasing temperature (or increasing pressure). Physically, the shrinkage of free volume upon cooling (or squeezing) enhances the cohesive energy among IMC molecules. Therefore, this amorphous drug becomes more rigid and achieves higher values of $X$. ECNLE predictions are quantitatively consistent with prior experiments utilizing Brillouin light scattering spectroscopy [48].

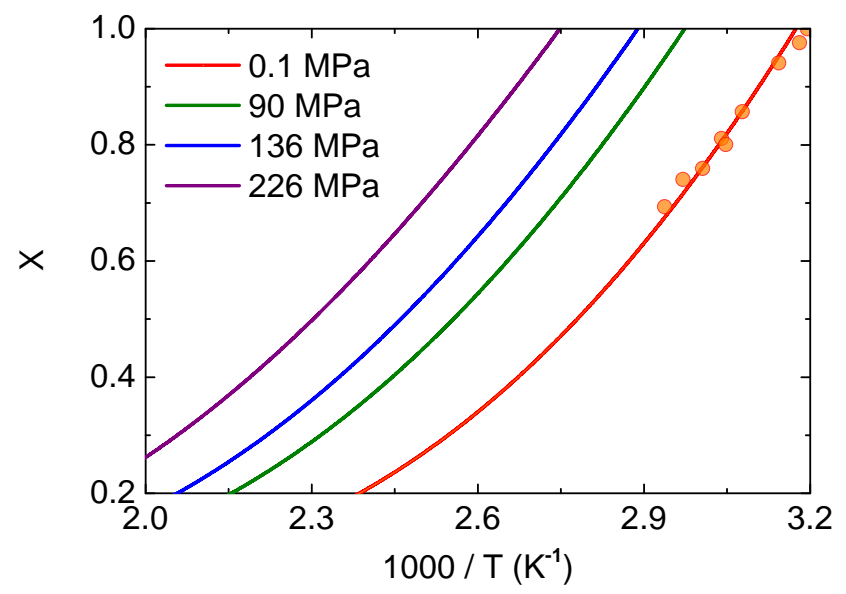

Fig. 5. (Color online) Dyre's shoving parameter of IMC as a function of inverse normalized temperature at different pressures. Solid lines and filled points represent ECNLE calculations and experimental data [48], respectively.

It is conspicuous that $X$ exhibits non-Arrhenius behaviors under isobaric conditions. The thermal sensitivity of $X$ is characterized by

$$
m_{X}=\left[\frac{\partial X}{\partial\left(T_{g} / T\right)}\right]_{T=T_{g}} .
$$

From Eqs. (17), (23), and (24), $m_{X}$ drops quickly by a factor of 1.36 between 0.1 and $260 \mathrm{MPa}$. Remarkably, Figure 6 shows a linear connection between $m_{X}$ and $m$. Our ECNLE theory yields $m=18.5 m_{X}$, which is quite close to Dyre's empirical rule $m=16 m_{X}$ [45-47]. Near the glass transition, the collective barrier overwhelms its local counterpart in the $\alpha$-relaxation process [2931]. Furthermore, Eq. (12) indicates that the elastic work is proportional to the instantaneous shear modulus. Thus, we acquire $\log _{10} \tau_{\alpha} \propto X$ and $m \propto m_{X}$ at $T \approx T_{g}$. In general, precise knowledge of $\tau_{\alpha}$ and $X$ can suggest a way of capturing molecular mobility via mechanical measurements $[49,50]$. 


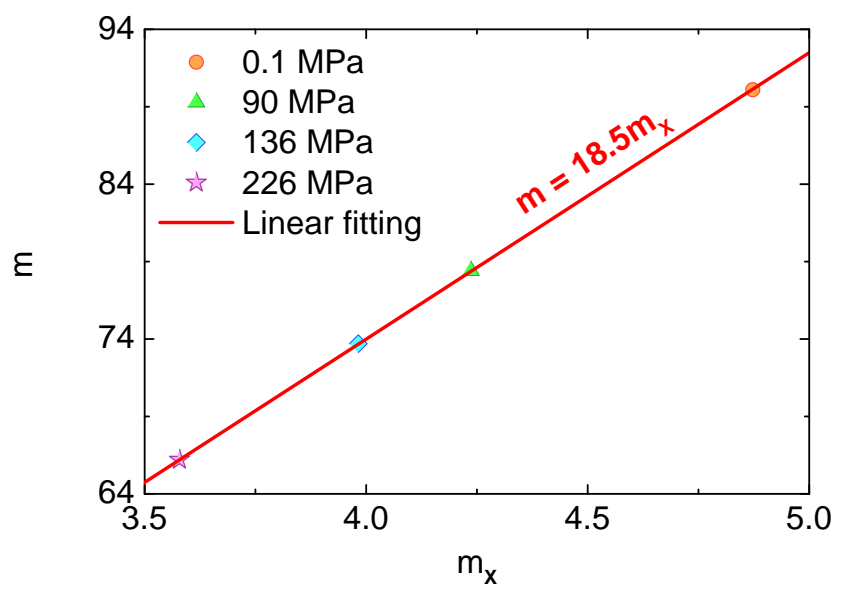

Fig. 6. (Color online) Correlations between $m_{X}$ and $m$ obtained from the ECNLE theory.

\section{CONCLUSIONS}

We have extended the ECNLE theory to consider the molecular mobility of IMC up to 226 MPa. Compression effects have been embedded in a chemical mapping from real IMC molecules to a hard-sphere fluid. This physical perspective allows us to determine the structural relaxation time, the dynamic fragility, and the shoving parameter at various thermodynamic conditions. At kinetic vitrification, we have found an important relation of $m \propto \beta T_{g} \propto m_{X}$. Our numerical calculations are in good accordance with previous experiments. Hence, the obtained results would improve understanding of the physical stability of IMC. Moreover, it is possible to develop our analytical approach to investigate the glassy dynamics in co-amorphous drugs.

\section{ACKNOWLEDGMENT}

This work was supported by the Vietnam National Foundation for Science and Technology Development (NAFOSTED) under grant number 103.01-2019.318.

\section{REFERENCES}

[1] R. J. Flower, Nat. Rev. Drug Discov. 2 (2003) 179.

[2] T. X. Xiang and B. D. Anderson, Mol. Pharm. 10 (2013) 102.

[3] X. Yuan, T. X. Xiang, B. D. Anderson, and E. J. Munson, Mol. Pharm. 12 (2015) 4518.

[4] S. Lucas, Headache 56 (2016) 436.

[5] D. W. Dodick, Curr. Pain Headache Rep. 8 (2004) 19.

[6] K. C. Sekar and K. E. Corff, J. Perinatol. 28 (2008) 60.

[7] H. Zhang, J. Fan, J. Wang, B. Dou, F. Zhou, J. Cao, J. Qu, Z. Cao, W. Zhao, and X. Peng, J. Am. Chem. Soc. 135 (2013) 17469.

[8] H. Zhang, J. Fan, J. Wang, S. Zhang, B. Dou, and X. Peng, J. Am. Chem. Soc. 135 (2013) 11663.

[9] X. Cao, T. Gao, J. Dong, X. Jiang, H. Zou, T. Liu, K. Yu, and W. Zeng, New J. Chem. 43 (2019) 7874.

[10] www.drugbank.ca/drugs/DB00328

[11] N. N. Shahin, N. F. Abdelkader, and M. M. Safar, Sci. Rep. 8 (2018) 1. 
[12] M. Rams-Baron, R. Jachowicz, E. Boldyreva, D. Zhou, W. Jamroz, and M. Paluch, Amorphous Drugs, Springer, Heidelberg (2018).

[13] B. C. Hancock and M. Parks, Pharm. Res. 17 (2000) 397.

[14] K. Grzybowska, S. Capaccioli, and M. Paluch, Adv. Drug Deliv. Rev. 100 (2016) 158.

[15] E. Kaminska, K. Adrjanowicz, D. Zakowiecki, B. Milanowski, M. Tarnacka, L. Hawelek, M. Dulski, J. Pilch, W. Smolka, I. Kaczmarczyk-Sedlak, and K. Kaminski, Pharm. Res. 31 (2014) 2887.

[16] S. Mohapatra, S. Samanta, K. Kothari, P. Mistry, and R. Suryanarayanan, Cryst. Growth Des. 17 (2017) 3142.

[17] Z. Wojnarowska, K. Adrjanowicz, P. Wlodarczyk, E. Kaminska, K. Kaminski, K. Grzybowska, R. Wrzalik, M. Paluch, and K. L. Ngai, J. Phys. Chem. B 113 (2009) 12536.

[18] A. D. Phan, J. Knapik-Kowalczuk, M. Paluch, T. X. Hoang, and K. Wakabayashi, Mol. Pharm. 16 (2019) 2992.

[19] A. D. Phan, K. Wakabayashi, M. Paluch, and V. D. Lam, RSC Adv. 9 (2019) 40214.

[20] A. D. Phan, T. T. T. Thuy, N. T. K. An, J. Knapik-Kowalczuk, M. Paluch, and K. Wakabayashi, AIP Adv. 10 (2020) 025128.

[21] A. D. Phan, A. Jedrzejowska, M. Paluch, and K. Wakabayashi, ACS Omega 5 (2020) 11035.

[22] A. D. Phan and K. Wakabayashi, Pharmaceutics 12 (2020) 177.

[23] J. P. Hansen and I. R. McDonald, Theory of Simple Liquids, Academic Press, London (2006).

[24] K. S. Schweizer and E. J. Saltzman, J. Chem. Phys. 119 (2003) 1181.

[25] K. S. Schweizer, J. Chem. Phys. 123 (2005) 244501.

[26] G. Ngele and J. Bergenholtz, J. Chem. Phys. 108 (1998) 9893.

[27] A. D. Phan and K. S. Schweizer, J. Phys. Chem. B 122 (2018) 8451.

[28] K. S. Schweizer, and G. Yatsenko, J. Chem. Phys. 127 (2007) 164505.

[29] S. Mirigian and K. S. Schweizer, J. Chem. Phys. 140 (2014) 194506.

[30] S. Mirigian and K. S. Schweizer, J. Chem. Phys. 140 (2014) 194507.

[31] S. Mirigian and K. S. Schweizer, Macromolecules 48 (2015) 1901.

[32] L. D. Landau and E. M. Lifshitz, Theory of Elasticity, Permagon Press, London (1975).

[33] S. P. Andersson and O. Andersson, Macromolecules 31 (1998) 2999.

[34] N. Dass and M. Kumari, Phys. Status Solidi (b) 124 (1984) 531.

[35] I. Avramov, A. Grzybowski, and M. Paluch, J. Non-Cryst. Solids 355 (2009) 733.

[36] M. Paluch, C. M. Roland, J. Gapinski, and A. Patkowski, J. Chem. Phys. 118 (2003) 3177.

[37] Z. Wojnarowska, K. Adrjanowicz, K. Kaminski, L. Hawelek, and M. Paluch, J. Phys. Chem. B 114 (2010) 14815.

[38] K. Adrjanowicz, K. Kaminski, Z. Wojnarowska, M. Dulski, L. Hawelek, S. Pawlus, M. Paluch, and W. Sawicki, J. Phys. Chem. B 114 (2010) 6579.

[39] K. Adrjanowicz, K. Kaminski, M. Paluch, and K. Niss, Cryst. Growth Des. 15 (2015) 3257.

[40] A. Patkowski, J. Gapinski, and G. Meier, Colloid Polym. Sci. 282 (2004) 874.

[41] C. M. Roland, S. Hensel-Bielowka, M. Paluch, and R. Casalini, Rep. Prog. Phys. 68 (2005) 1405.

[42] R. Casalini and C. M. Roland, Phys. Rev. B 71 (2005) 014210.

[43] M. Paluch, E. Masiewicz, A. Grzybowski, S. Pawlus, J. Pionteck, and Z. Wojnarowska, J. Chem. Phys. 141 (2014) 134507.

[44] E. Masiewicz, A. Grzybowski, A. P. Sokolov, and M. Paluch, J. Phys. Chem. Lett. 3 (2012) 2643.

[45] T. D. Cuong, A. D. Phan, K. Wakabayashi, and P. T. Huy, J. Non-Cryst. Solids 538 (2020) 120024.

[46] J. C. Dyre, J. Non-Cryst. Solids 235 (1998) 142.

[47] C. Klieber, T. Hecksher, T. Pezeril, D. H. Torchinsky, J. C. Dyre, and K. A. Nelson, J. Chem. Phys. 138 (2013) $12 \mathrm{~A} 544$.

[48] K. L. Kearns, T. Still, G. Fytas, and M. D. Ediger, Adv. Mater. 22 (2010) 39.

[49] T. Hecksher and J. C. Dyre, J. Non-Cryst. Solids 407 (2015) 14.

[50] T. Hecksher, D. H. Torchinsky, C. Klieber, J. A. Johnson, J. C. Dyre, and K. A. Nelson, Proc. Natl. Acad. Sci. U.S.A. 114 (2017) 8710. 